\title{
Effects of asynchrony on rabbit blastocyst development
}

\author{
B. Fischer \\ Department of Anatomy and Reproductive Biology, Medical Faculty, RWTH Aachen, \\ D-5100 Aachen, Federal Republic of Germany
}

\begin{abstract}
Summary. The development of synchronously and asynchronously transferred rabbit morulae (recovered at Day 3 p.c.) and blastocysts (recovered at Day 4 p.c.) was investigated before the anticipated time of implantation. The results obtained with various techniques (evaluation of gross morphology, measurement of diameter, thymidine incorporation, light and electron microscopy) led basically to the same conclusions. Embryos being asynchronously transferred to the uterus of recipient rabbits survived, at least in terms of certain cellular functions like cell proliferation, for more than 2 days. Development, however, was clearly retarded and ultrastructural examination revealed substantial cell damage. Some blastocyts showed, even after 3 days, normal growth and cell proliferation indicating considerable differences between individuals in the ability to compensate for suboptimal developmental conditions before implantation. In general, this ability was greater in the transferred Day-3 morulae than in the Day- 4 blastocysts. Embryonic growth and the ability to dissolve the zona pellucida, to synthesize crystalloid bodies and to differentiate extraembryonic endoderm indicated the maintenance of some developmental functions under asynchronous conditions.

Blastocyst development was influenced by the progestational stage of the recipient. At 1 day after transfer into asynchronous older uteri, blastocyst diameters were larger and cell proliferation was increased compared with all other groups, suggesting an attempt of the blastocyst to adjust to the more advanced maternal milieu. Development in asynchronous younger uteri was delayed.

No comparable differences in development were found in cultured embryos for which the media had been supplemented with flushings from the same progestational uterine stages as used for transfer. Thymidine incorporation in cultured embryos did not differ between the various supplements $(P>0.05)$ and was generally lower than in chronologically aged asynchronously transferred embryos $(P<0.05$ for Day-3 and $P<0.001$ or $P>0.05$ for Day-4 embryos).
\end{abstract}

Keywords: uterine transfer; asynchrony; blastocyst; rabbit preimplantation development; in-vitro culture

\section{Introduction}

Increasing attention is paid to preimplantation embryos developing under unphysiological conditions. A typical example for an unphysiological uterine milieu is that of asynchrony, i.e. in which the stage of embryo development is discrepant from that of endometrial transformation and secretion. Such conditions are created, for example, by common infertility therapies involving hormone treatment of the mother or asynchronous embryo transfer, both in the human and in livestock.

Chang (1950), by performing asynchronous embryo transfers in the rabbit, was the first to prove the need for close synchronization of donor and recipient; transferred rabbit embryos survived only if asynchrony between embryonic and maternal development did not exceed 1 or 2 
day(s). Compared with primates the demand for synchrony is extremely strict in the rabbit. The results of Chang (1950) were mainly based on the rate of implantation and survival to term. The present study was performed to investigate development of asynchronously transferred embryos before implantation. The aim of the study was to define the time interval during which embryos can survive asynchronous conditions and to characterize development under such conditions to learn more about the ability of preimplantation embryos to compensate for suboptimal developmental conditions. Additionally, development of cultured embryos was studied. The culture medium had been supplemented with flushings from the same progestational uteri as used for transfer.

\section{Materials and Methods}

\section{Embryos, embryo transfer, in-vitro culture}

The housing of the rabbits, the superovulation treatment of the donors with FSH-P (Burns Biotec, Omaha, NE, USA) and embryo recovery have been previously described in detail by Fischer \& Meuser-Odenkirchen (1988). Donors were killed 3 and 4 days after mating (p.c.) and injection of hCG (Prolan: Bayer, Leverkusen, FRG) to recover morulae and blastocysts, respectively. Only embryos of normal morphology were used for the study. Embryos from various donors were pooled and randomly divided amongst the experimental groups.

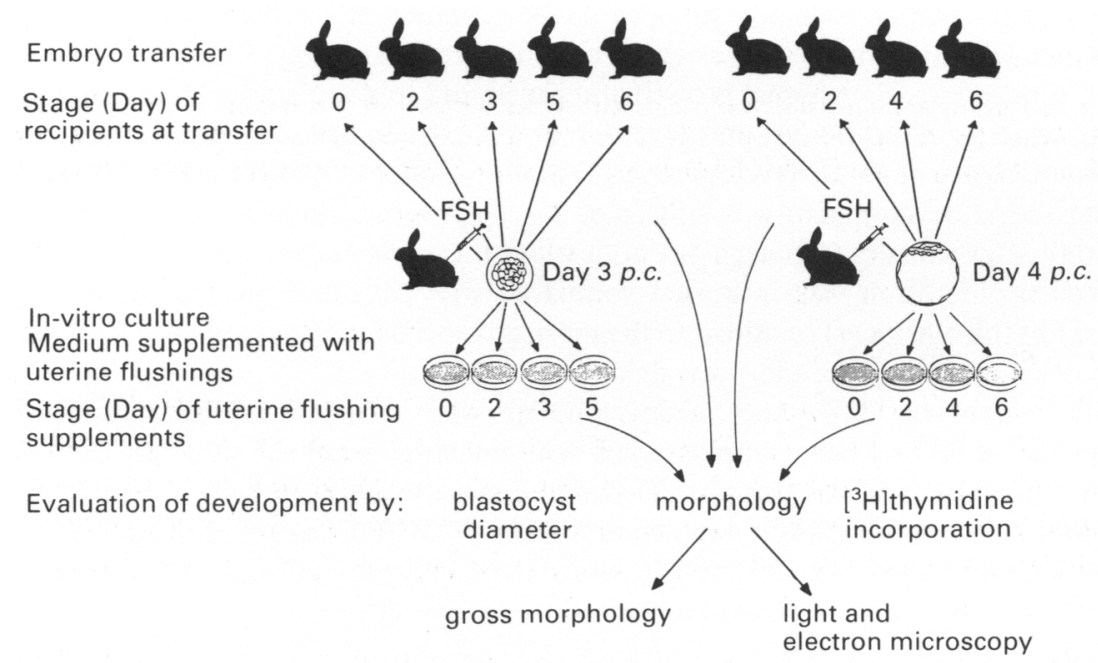

Fig. 1. Experimental design.

Compared with the mating of the donors, the injection of hCG to the recipients $(\mathrm{N}=18$ for Day- 3 morulae and $\mathrm{N}=21$ for Day-4 blastocysts) was delayed by 3-4 h to compensate for the possible developmental retardation of the embryos due to the transfer manipulations. In total, 1123 embryos were used for the transfer experiments. The experimental groups chosen are illustrated in Fig. 1. Embryos were transferred into uteri with double ligations by transmural puncture as described by Fischer (1987). On average, 29 embryos were transferred per female (Day-3 morulae, mean $=33$; Day-4 blastocysts, mean $=25$ ). The embryos were recovered $1-3$ day $(s)$ after transfer, using the same flushing procedure as before transfer. After removal of the ligatures the embryos could be easily recovered. Exceptions were the Day- 4 blastocysts transferred for 3 days which might have been already attached to the endometrium at that time. At autopsy an average of 8.3 corpora lutea of normal gross morphology were found per recipient.

Medium BSM II (Maurer, 1978) containing $0 \cdot 1 \%$ BSA was used for flushing, examination, pooling, storage and transfer of the embryos. For in-vitro culture Ham's F-10 medium (Ham, 1963) was supplemented with uterine flushings acquired from non-pregnant or pseudopregnant rabbits (see Fig. 1). Details of culture media and culture technique were as previously given (Fischer, 1987). The protein concentrations of the media ranged from $0.7 \mathrm{mg} / \mathrm{ml}$ at Day 0 to $4.8 \mathrm{mg} / \mathrm{ml}$ if flushings of Day-6 pseudopregnant rabbits were used (Fischer, 1988). Altogther, 279 embryos were cultured for 2 days. 


\section{Gross morphology, diameter, thymidine incorporation}

All embryos were examined for gross morphology; totally degenerated embryos were not processed for any further evaluation. The vitelline diameter, i.e. the diameter within opposite extracelullar coverings such as the zona pellucida, was measured with an ocular micrometer (Jung \& Fischer, 1988). Incubation with tritiated thymidine (5 $\mu \mathrm{Ci} / \mathrm{ml}$; sp.act. $5 \mathrm{Ci} / \mathrm{mmol}$ or $185 \mathrm{GBq} / \mathrm{mmol}$; Amersham Buchler, Braunschweig, FRG) for $4 \mathrm{~h}$ and subsequent processing for liquid scintillation counting (tissue homogenizer, Lumasolve; scintillation fluid, Lipoluma: Lumac, Schaesberg, The Netherlands) was performed as previously described (Fischer, 1987; Schumacher \& Fischer, 1988). For quality control unlabelled embryos were included; the results for these did not differ from background (i.e. values for the scintillation fluid). Compared with values from labelled embryos, background was minimal and was therefore not subtracted from incorporation data. Results are expressed as d.p.m. (disintegrations per minute) per embryo.

\section{Light and electron microscopy}

In total, 27 Day-3 morulae, recovered 1-3 day(s) after transfer, were processed for light and electron microscopy. Electron microscopical examination was performed on 12 embryos which had been recovered 2 days after transfer. The interval between recovery and fixation did not exceed $20 \mathrm{~min}$. Embryos were fixed in $2.5 \%$ glutaraldehyde in $0.1 \mathrm{M}$-cacodylate buffer $(290 \mathrm{mosmol}, \mathrm{pH} 7.4)$ for $1 \mathrm{~h}$, rinsed in $0.1 \mathrm{M}$-cacodylate buffer and post-fixed in $2 \%$ cacodylate-buffered $\mathrm{OsO}_{4}$ for $1 \mathrm{~h}$. Dehydration was performed in a graded series of ethanols to propylene oxide, during which block staining with $1 \%$ uranyl acetate in $75 \%$ ethanol was carried out. Semithin sections of the araldite (Araldit M: Merck, Darmstadt, FRG) -embedded embryos were cut serially, stained with azur II-methylene blue and assessed and photographed with an Axiomat NDC (Zeiss, Oberkochen, FRG). Ultrathin sections were mounted on Formvar-coated copper grids, stained with lead citrate and uranyl acetate and examined with a Zeiss EM 10 electron microscope at $60-80 \mathrm{kV}$.

\section{Statistical analysis}

Diameter and incorporation data were examined by the Kruskal-Wallis non-parametric H-test with multiple comparisons. When this test indicated statistically significant differences, individual means were pairwise compared using the Mann-Whitney U-test (Sachs, 1984). In all other cases Student's $t$ tests or $\chi^{2}$ tests were applied.

\section{Results}

\section{Recovery and gross morphology}

Overall, $58 \%$ of the transferred embryos were recovered. Recovery was slightly higher for Day4 blastocysts $(P>0.05$; Table 1$)$. Within both ages consistently better recoveries were obtained if the embryos had been transferred synchronously or into comparatively more advanced uteri (Table 1). Compared with recovery 1 and 2 day(s) after transfer, recovery after 3 days yielded statistically significantly fewer embryos in both ages (3rd day $v$ s 2 nd day: $P<0.05$, vs 1st day: $P<0.01$; Table

Table 1. Rate of recovery of synchronously and asynchronously transferred rabbit embryos

\begin{tabular}{lccccc}
\hline & \multicolumn{4}{c}{ Embryonic age at transfer } \\
\cline { 2 - 3 } $\begin{array}{l}\text { Stage of recipients } \\
\text { at transfer } \\
\text { (days after hCG) }\end{array}$ & $\begin{array}{c}\text { No. } \\
\text { transferred }\end{array}$ & $\begin{array}{c}\text { No. } \\
\text { recovered }(\%)\end{array}$ & & $\begin{array}{c}\text { No. } \\
\text { transferred }\end{array}$ & $\begin{array}{c}\text { No. } \\
\text { recovered (\%) }\end{array}$ \\
\cline { 2 - 3 } \cline { 5 - 6 } & 110 & $27(25)^{\mathrm{a}}$ & & 86 & $39(45)^{\mathrm{a}}$ \\
2 & 138 & $67(49)^{\mathrm{ab}}$ & & 186 & $80(43)^{\mathrm{a}}$ \\
3 & 81 & $50(62)^{\mathrm{b}}$ & & - & \\
4 & - & $61(71)^{\mathrm{b}}$ & & -126 & $98(78)^{\mathrm{b}}$ \\
5 & 86 & $133(72)^{\mathrm{b}}$ & & 125 & $93(74)^{\mathrm{b}}$ \\
6 & 185 & $338(56)$ & & 523 & $310(59)$ \\
\hline
\end{tabular}

Values with different superscripts within vertical rows are statistically different $(P<0.001)$. 


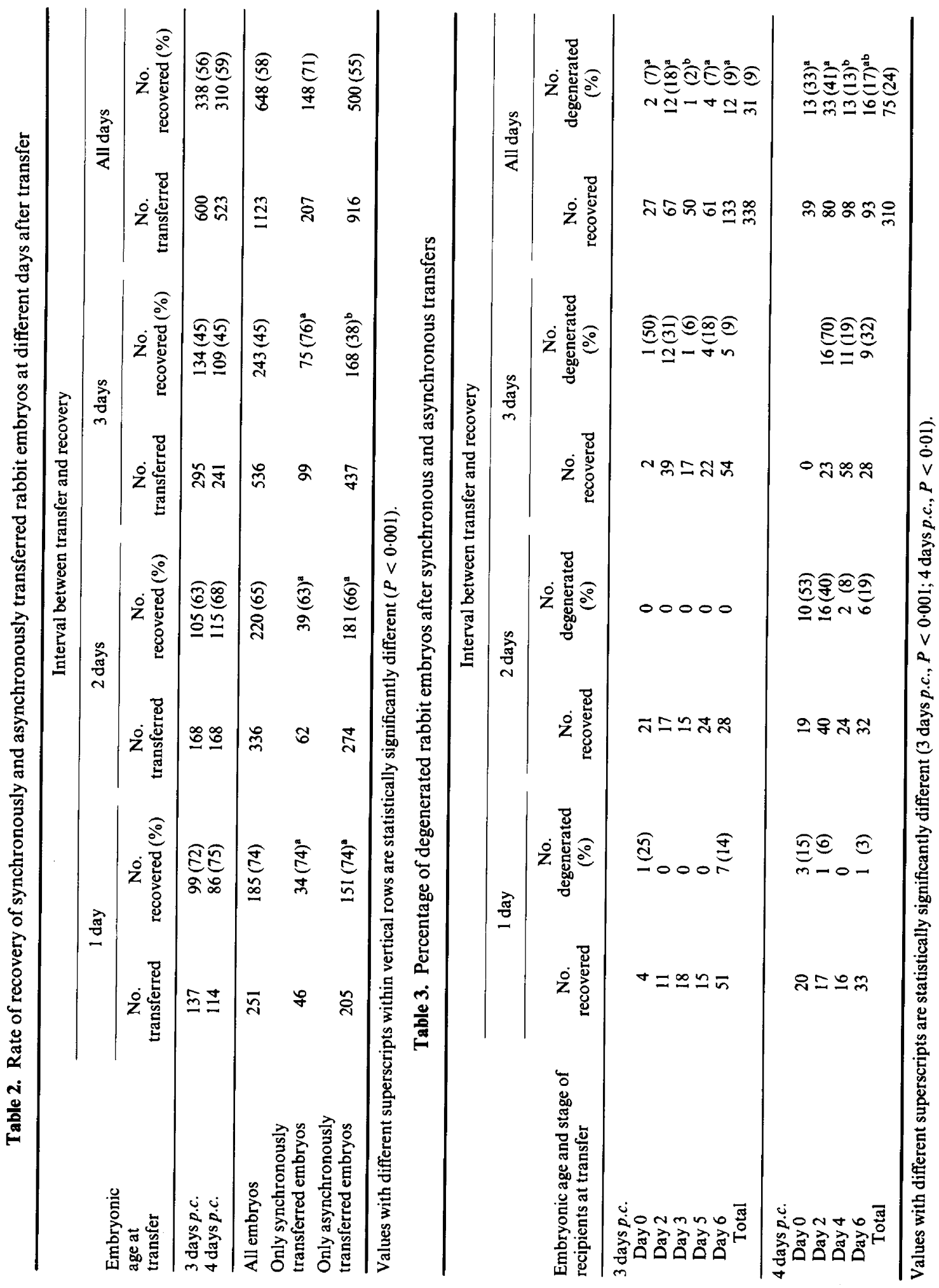



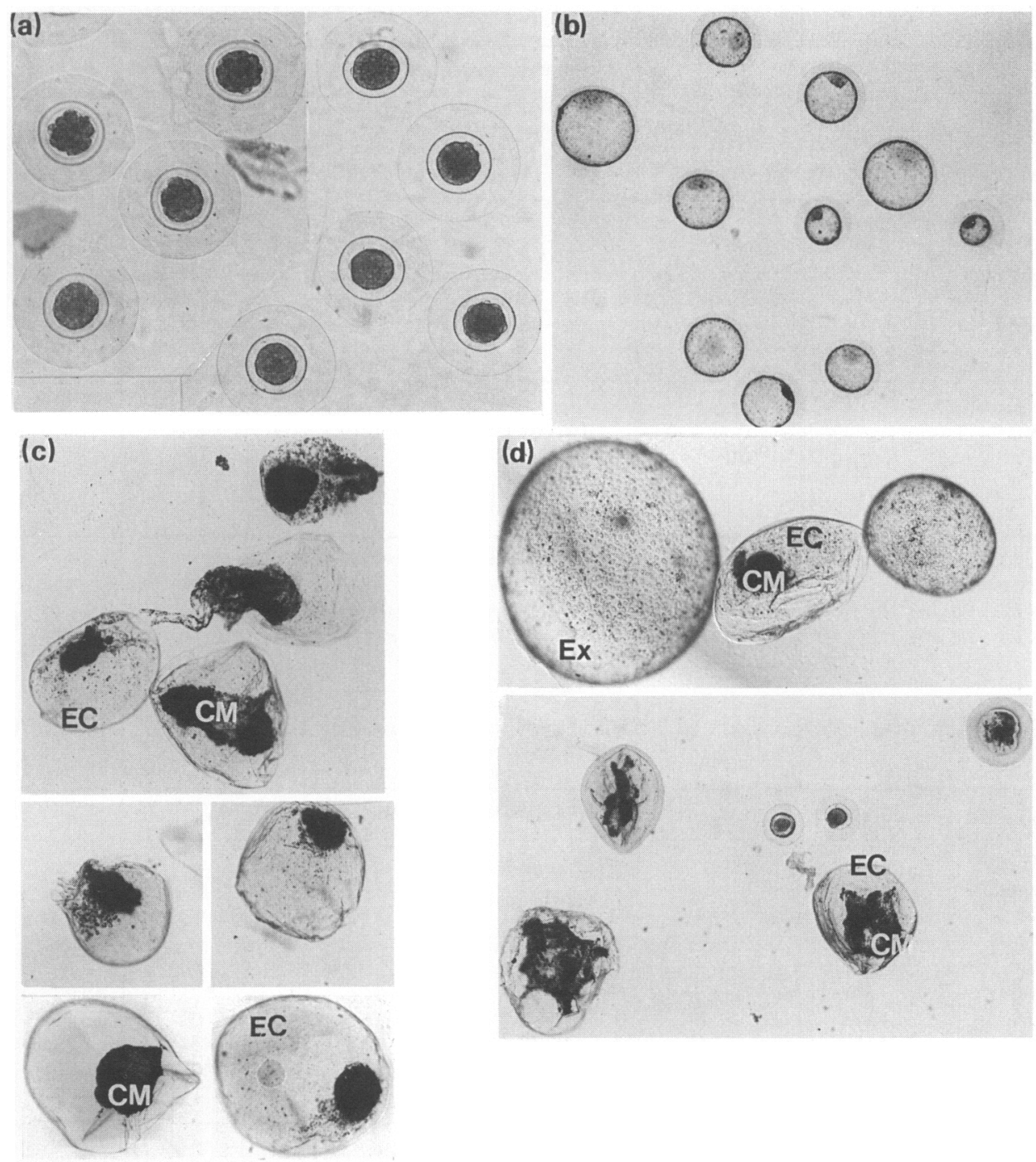

Fig. 2. Gross morphology of rabbit embryos before and after transfer: (a) morulae recovered 3 days $p . c$. , before transfer $(\times 46)$; (b) blastocysts recovered 4 days p.c., before transfer $(\times 17)$; (c) asynchronously transferred Day-4 blastocysts: recovery 2 days after transfer into a Day-2 pseudopregnant recipient $(\times 17)$; EC, extracellular covering, $\mathbf{C M}$, degenerated cellular material; (d) asychronously transferred Day-4 blastocysts: recovery 3 days after transfer into a Day-2 pseudopregnant recipient $(\times 17)$; Ex, expanded blastocyst, EC, extracellular covering, $\mathrm{CM}$, degenerated cellular material.

2). Recovery was not reduced until 3 days after asynchronous transfer $(P<0.001)$; by then more than half of the transferred embryos were no longer recoverable and seemed to have disintegrated. Apart from coverings like empty zonae pellucidae no other embryonic remnants were detectable in the flushings. This finding is supported by the percentage of degenerated embryos at recovery (Table 3). Although this figure was already increased for Day-4 blastocysts by 2 days after transfer, the overall rate of degeneration was clearly enhanced after 3 days, particularly for Day-4 blastocysts (49\% compared with $19 \%$ in Day-3 morulae). For embryos of both ages degeneration was 
Table 4. Diameters of synchronously and asynchronously transferred rabbit embryos (in mm)

\begin{tabular}{|c|c|c|c|c|c|c|c|c|c|c|c|c|}
\hline \multirow{3}{*}{$\begin{array}{l}\text { Embryonic age } \\
\text { and stage of } \\
\text { recipients at transfer }\end{array}$} & \multicolumn{12}{|c|}{ Interval between transfer and recovery } \\
\hline & \multicolumn{4}{|c|}{1 day } & \multicolumn{4}{|c|}{2 days } & \multicolumn{4}{|c|}{3 days } \\
\hline & Mean & s.d. & $\mathrm{CV} \%$ & $n$ & Mean & s.d. & CV\% & $n$ & Mean & s.d. & $\mathrm{CV} \%$ & $n$ \\
\hline \multicolumn{13}{|l|}{3 days p.c. } \\
\hline Day 0 & - & & & & $0.56^{\mathrm{a}}$ & $0 \cdot 14$ & 25 & 18 & - $^{*}$ & & & \\
\hline Day 2 & $0.40^{\mathrm{a}}$ & $0 \cdot 12$ & 30 & 8 & $0.54^{\mathrm{a}}$ & 0.15 & 28 & 9 & $1.74^{\mathrm{a}}$ & 0.49 & 28 & 20 \\
\hline Day 3 & $0.37^{\mathrm{ab}}$ & 0.10 & 27 & 10 & $1.01^{\mathrm{b}}$ & 0.05 & 5 & 5 & $2 \cdot 14^{b}$ & $0 \cdot 30$ & 14 & 8 \\
\hline Day 5 & $0.29^{\mathrm{b}}$ & $0 \cdot 10$ & 34 & 13 & —* $^{*}$ & & & & $0.99^{\mathrm{c}}$ & 0.26 & 26 & 8 \\
\hline Day 6 & 一 $^{*}$ & & & & -* & & & & $0.64^{\mathrm{c}}$ & $0 \cdot 23$ & 36 & 8 \\
\hline \multicolumn{13}{|l|}{4 days $p . c$. } \\
\hline Day 0 & $0.78^{\mathrm{a}}$ & 0.13 & 17 & 10 & $1.00^{\mathrm{a}}$ & $0 \cdot 10$ & 10 & 4 & —* & & & \\
\hline Day 2 & $0.51^{\mathrm{b}}$ & $0 \cdot 10$ & 20 & 13 & $0.98^{\mathrm{a}}$ & 0.23 & 23 & 8 & $1 \cdot 38^{\mathrm{a}}$ & $0 \cdot 66$ & 48 & 4 \\
\hline Day 4 & $0.98^{c}$ & 0.20 & 20 & 13 & $2 \cdot 74^{\mathrm{b}}$ & 0.69 & 25 & 20 & $3 \cdot 51^{b}$ & 1.07 & 30 & 8 \\
\hline Day 6 & $1 \cdot 26^{\mathrm{d}}$ & 0.27 & 21 & 19 & $1 \cdot 15^{\mathrm{a}}$ & 0.61 & 53 & 7 & $1.92^{\mathrm{a}}$ & 1.48 & 77 & 6 \\
\hline
\end{tabular}

CV\%, coefficient of variation (s.d. divided by the mean $\times 100$ ); $n$, number of embryos.

*No measurements.

3 days p.c.: 1st day: ${ }^{\text {ab }} P<0.05$; 2nd day: ${ }^{\text {ab }} P<0.001 ; 3$ rd day: ${ }^{\text {ab }} P<0.05$; ${ }^{\text {ab,ac,bc }} P<0.001$.

4 days $p . c .: 1$ st day: ${ }^{\text {ac }} P<0.05 ;{ }^{\text {ab }} P<0.01$; ${ }^{\text {ad,bc.bd,cd } P}<0.001 ; 2$ nd day: ${ }^{\text {ab }} P<0.001$; 3 rd day: ${ }^{\text {ab }} P<0.05$.

Table 5. Thymidine incorporation (d.p.m./embryo) in synchronously and asynchronously transferred rabbit embryos

\begin{tabular}{|c|c|c|c|c|c|c|c|c|c|c|c|c|}
\hline \multirow{3}{*}{$\begin{array}{l}\text { Embryonic age } \\
\text { and stage of } \\
\text { recipients at } \\
\text { transfer }\end{array}$} & \multicolumn{12}{|c|}{ Interval between transfer and recovery } \\
\hline & \multicolumn{4}{|c|}{1 day } & \multicolumn{4}{|c|}{2 days } & \multicolumn{4}{|c|}{3 days } \\
\hline & Mean & s.d. & $\mathrm{CV} \%$ & $n$ & Mean & s.d. & $\mathrm{CV} \%$ & $n$ & Mean & s.d. & $\mathrm{CV} \%$ & $n$ \\
\hline \multicolumn{13}{|l|}{3 days $p$} \\
\hline Day 0 & 一 $^{*}$ & & & & $4921^{\mathrm{z}}$ & 594 & 42 & 19 & -** & & & \\
\hline Day 2 & $4249^{a}$ & 411 & 19 & 8 & $8125^{b}$ & 1367 & 63 & 14 & $131703^{\mathrm{a}}$ & 13915 & 52 & 25 \\
\hline Day 3 & $3141^{b}$ & 638 & 45 & 13 & $25105^{\mathrm{c}}$ & 4090 & 49 & 11 & $163743^{a}$ & 27980 & 66 & 15 \\
\hline Day 5 & $3969^{c}$ & 781 & 48 & 13 & $12688^{d}$ & 2752 & 53 & 17 & $23740^{b}$ & 7541 & 105 & 15 \\
\hline Day 6 & $1908^{d}$ & 117 & 22 & 35 & $5221^{\circ}$ & 1362 & 69 & 20 & $10511^{b}$ & 1551 & 99 & 45 \\
\hline \multicolumn{13}{|l|}{4 days p.c. } \\
\hline Day 0 & $14465^{\mathrm{a}}$ & 1221 & 29 & 15 & $12699^{\mathrm{a}}$ & 3250 & 72 & 9 & —* & & & \\
\hline Day 2 & $6309^{\mathrm{a}}$ & 910 & 56 & 15 & $11089^{2}$ & 2458 & 99 & 22 & $51376^{a}$ & 23019 & 119 & 7 \\
\hline Day 4 & $29815^{\mathrm{b}}$ & 2834 & 33 & 16 & $245957^{b}$ & 37892 & 72 & 22 & $167710^{\mathrm{a}}$ & 31629 & 96 & 37 \\
\hline Day 6 & $40259^{c}$ & 4331 & 43 & 32 & $47974^{2}$ & 8276 & 75 & 26 & $89454^{a}$ & 21309 & 79 & 15 \\
\hline
\end{tabular}

$\mathrm{CV} \%$, coefficients of variation (s.d. divided by the mean $\times 100$ ); $n$, number of embryos.

*No measurements.

3 days p.c.: 1st day: ${ }^{\text {ab }} P<0.05$; ${ }^{\text {bd }} P<0.01$; ad,od $P<0.001$; 2nd day: ${ }^{\text {bd }} P<0.05$; ac,bc,dc,ec,ac $P<0.001$; 3rd day: ab $P<0.001$.

4 days $p . c .: 1$ st day: ${ }^{\text {bc } P}<0.01 ;$ ab,ac $P<0.001 ; 2$ nd day: ${ }^{\text {ab }} P<0.001$.

notably higher in asynchronously transferred embryos. Judged by the rate of recovery and percentage of degeneration most embryos tolerated 2 days of asynchrony, and some even 3 days. From all groups studied the poorest results concerning recovery and degeneration were achieved after transfer into non-pregnant/non-pseudopregnant recipients and, for Day-4 blastocysts, into the less advanced Day-2 uteri.

The gross morphology before and after transfer is illustrated in Fig. 2. The morphology of the degenerated asynchronously transferred embryos at recovery did not allow an estimation of how 
Day-3 morulae

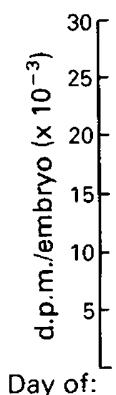

(a)

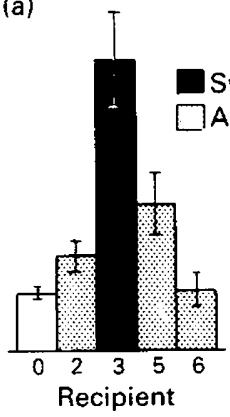

(b)

Synchronous

Asynchronous

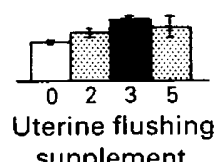

Day-4 blastocysts

(a)

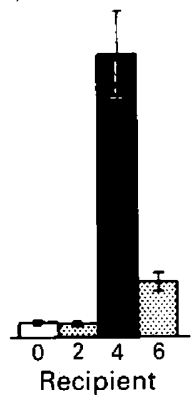

(b)

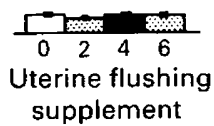

Fig. 3. Thymidine incorporation in (a) transferred (recovered 2 days later) and (b) cultured (for 2 days) rabbit embryos. Values are mean \pm s.e.m. For numbers of transferred embryos see Table 5; numbers of cultured Day-3 morulae are 18,11, 46, 16 for Day $0,2,3,5$, of cultured Day-4 blastocysts $23,37,91,27$ for Day $0,2,4$ and 6, respectively.

long the embryos had survived in the asynchronous uteri. Embryos as degenerate as the ones shown in Figs 2(c) and (d) were not further considered for analyses (see 'Materials \& Methods').

\section{Diameter, thymidine incorporation}

According to the progestational stage of the recipient the size and the cell proliferation of the transferred embryos differed markedly (Tables 4 \& 5). Compared with synchronously transferred embryos impairment of development of asynchronously transferred embryos is clearly shown by smaller diameters and lower incorporation data. Synchronously transferred embryos corresponded with chronologically aged non-transferred controls in size and thymidine incorporation, indicating no detrimental effect of the manipulations associated with the embryo transfer. The control data for the diameter (mean \pm s.d., number of embryos) were $0.11 \pm 0.01(n=45)$ at Day 3 p.c., $0.39 \pm 0.07(n=25)$ at Day 4 and $1.21 \pm 0.25(n=20)$ at Day 5 (all comprisons; $P<0.001)$; data for thymidine incorporation of control embryos are given by Fischer (1987).

For Day-4 blastocysts specific influences of the progestational stage of the recipient were detectable: at 1 day after transfer the blastocysts which had been transferred into the more advanced Day-6 uteri were clearly more expanded and had a statistically significantly increased thymidine incorporation compared with all other groups. In contrast, transfers into less advanced uteri were associated with a retardation in development (Tables 4 \& 5).

The superior conditions for development in a synchronous uterine milieu became manifest for embryos of both ages not earlier than 2 days after transfer (Tables 4 \& 5). A transfer arrangement known to be compatible with further development (Chang, 1950) was studied for 3-day-old morulae being transferred into Day- 2 recipients. Judged by thymidine incorporation these embryos caught up with the synchronous ones as late as 3 days after transfer $(P>0.05$; Table 5$)$.

Even after 3 days in an asynchronous uterine milieu some blastocysts showed a remarkably high level of thymidine incorporation. High variations in incorporation data were found at that day (Table 5). For example, at 3 days after transfer of Day-4 blastocysts into Day-2 recipient uteri incorporation data varied from 10000 to 180000 d.p.m./embryo, indicating a considerably divergent ability for compensation for unphysiological developmental conditions between individual blastocysts.

Supplementation of the culture medium with synchronous or asynchronous uterine flushings did not alter the cell proliferation of the cultured Day-3 or Day-4 embryos significantly $(P>0.05$; Fig. 3). Therefore, the uterine components determining synchrony in vivo were not effective in vitró. Besides quantitative and qualitative deficiencies, e.g. in relation to the continuous dynamics in 

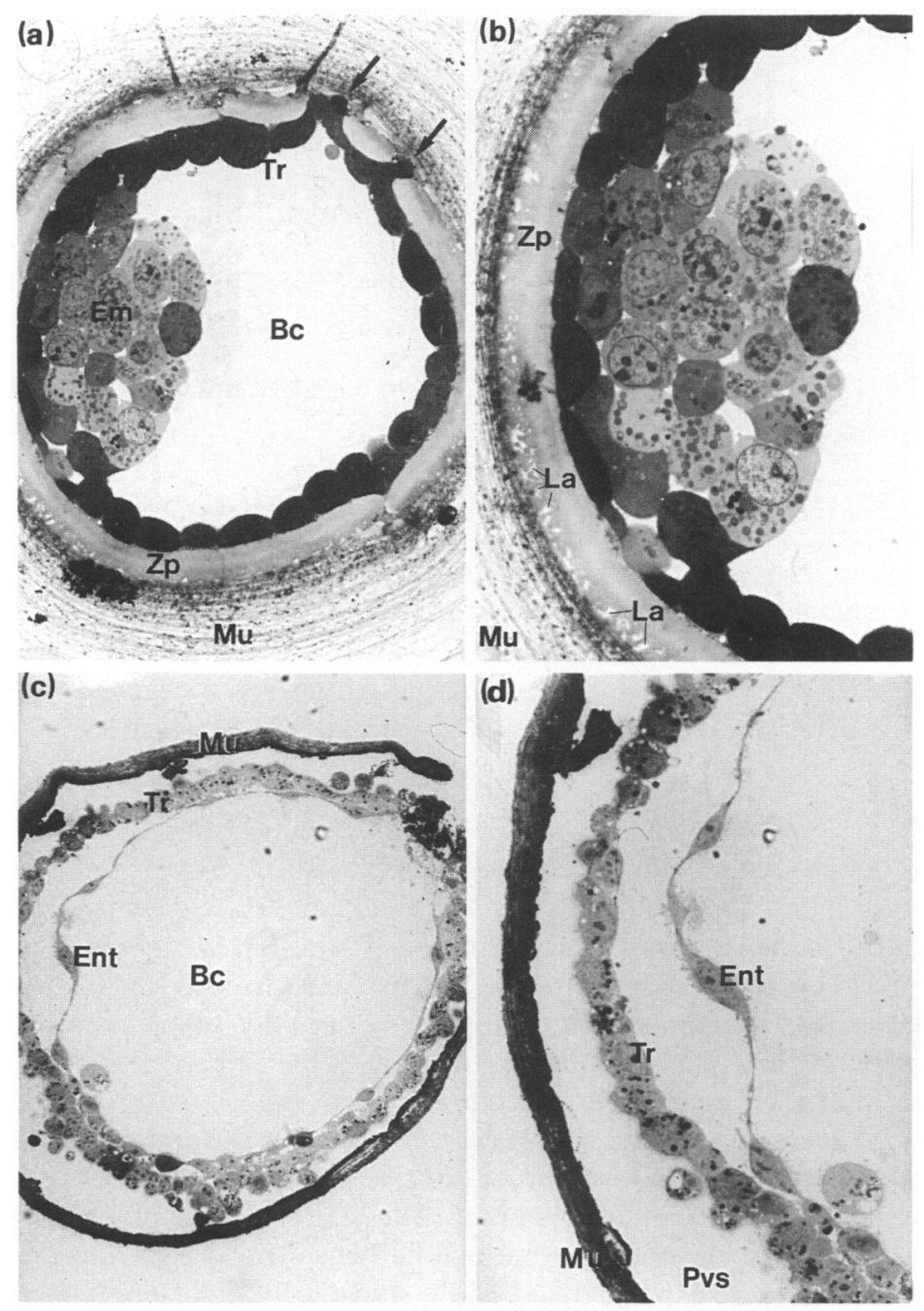

Fig. 4. Semithin sections of asynchronously transferred Day-3 rabbit morulae. (a, b) Recovery 1 day after transfer into a non-pregnant/non-pseudopregnant recipient. (a) The morula has developed into a slightly expanded blastocyst with the embryoblast cells (Em) bulging into the blastocyst cavity $(\mathrm{Bc})$. The dark staining trophoblast cells $(\mathrm{Tr})$ are surrounded by a broken, but otherwise more or less entirely preserved zona pellucida $(\mathrm{Zp})$. In small, localized areas trophoblast cells perforate the zona pellucida (arrows). $\times 357$. (b) While the inner surface of the zona pellucida $(\mathrm{Zp})$ shows a homogeneous appearance, the outer seems to be in the process of dissolution as indicated by many lacunar erosions $(\mathrm{La})$. Mu, mucoprotein layer. $\times 590$. (c, d) Recovery 3 days after transfer into a Day-6 pseudopregnant recipient. (c) The trophoblast cells (Tr) are entirely covered by extraembryonic endoderm cells (Ent) towards the blastocyst cavity (Bc). Mu, mucoprotein layer. $\times 120$. (d) The zona pellucida is completely dissolved; no remnants are visible within the perivitelline space (Pvs). The blastocyst's covering is a darkly stained mucoprotein layer $(\mathrm{Mu})$. Tr, trophoblast; Ent, extraembryonic endoderm. $\times 303$.

synthesis and release of these components, direct cellular contact was missing in culture and may be crucial in mediating synchrony. The comparison of chronologically aged cultured and asynchronously transferred embryos in Fig. 3 indicates a generally lower thymidine incorporation in cultured embryos, especially for the Day-3 morulae $(P<0.05)$. 

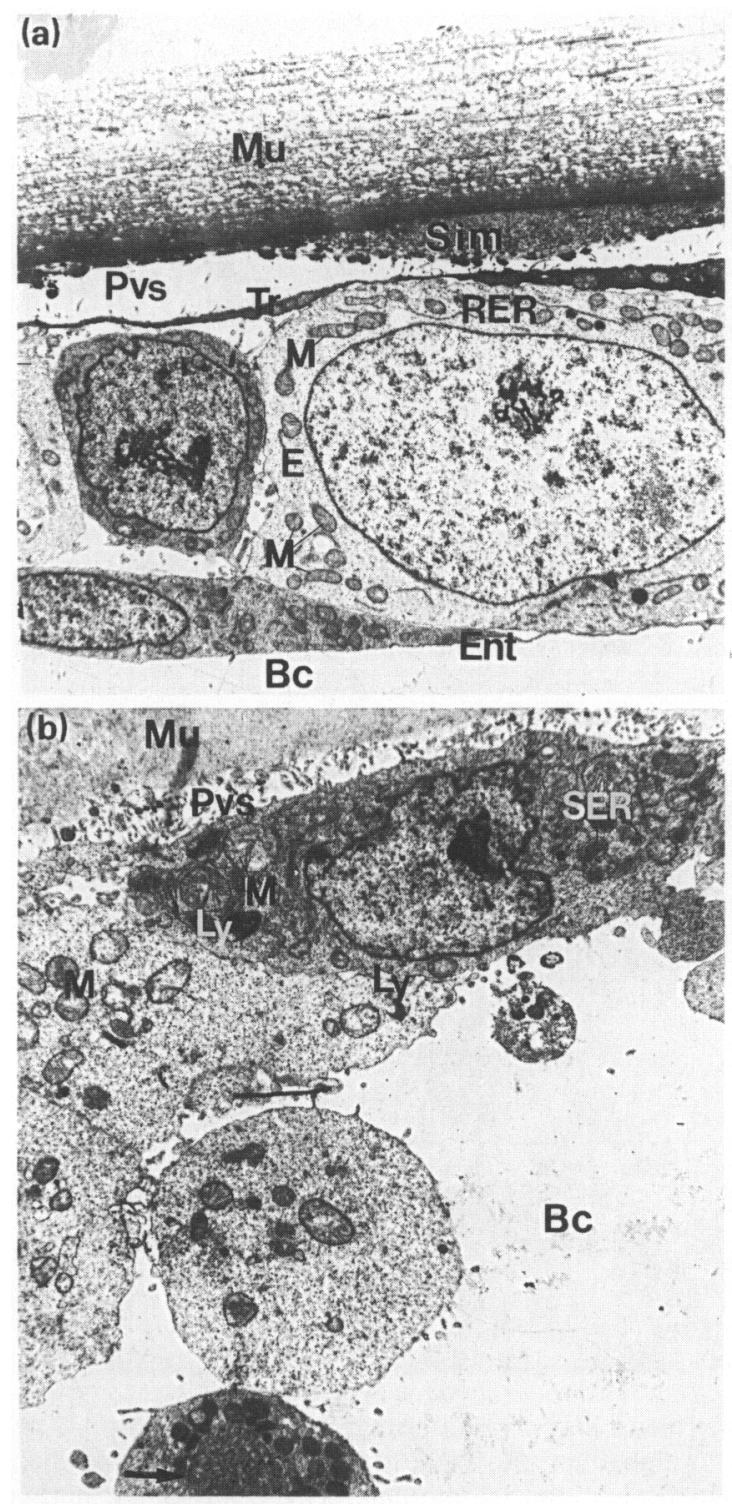

Fig. 5. TEM micrographs of synchronously and asynchronously transferred Day-3 rabbit morulae recovered 2 days after transfer. (a) After transfer into a Day-3 pseudopregnant recipient (synchronous transfer). The embryoblast cells (E) whose organelles are characterized by a typical stage-specific ultrastructure (M, circular to ovoid shaped mitochondria, RER, rough endoplasmic reticulum) are covered towards the blastocyst cavity $(\mathrm{Bc})$ by flattened endoderm (Ent), towards the perivitelline space (Pvs) by densely stained, thin trophoblast cells ( $\mathrm{Tr}$; Rauber cells). Occasionally cells with an electron-dense cytoplasm were found within the embryoblast cells. The blastocyst is covered by the mucoprotein layer $(\mathrm{Mu})$ with its characteristically condensed inner part. The super-imposed material ( $\mathrm{Sim}$ ) within the perivitelline space may be first traces of the neozona. $\times 3810$. (b) After transfer into a non-pregnant/non-pseudopregnant recipient. Developmental retardation is indicated by the persistence of smooth endoplasmic reticulum (SER) which should have been replaced by rough one at this age (Merchant, 1970; own results). Signs of degeneration are visible, e.g. vacuolated mitochondria $(M)$ with peripherally displaced cristae or detached cells, often with condensed chromatin (arrow), and cell remnants in the blastocyst cavity $(\mathrm{Bc})$. Ly, secondary lysosomes, Pvs, perivitelline space, $\mathrm{Mu}$, mucoprotein layer. $\times 4770$. 

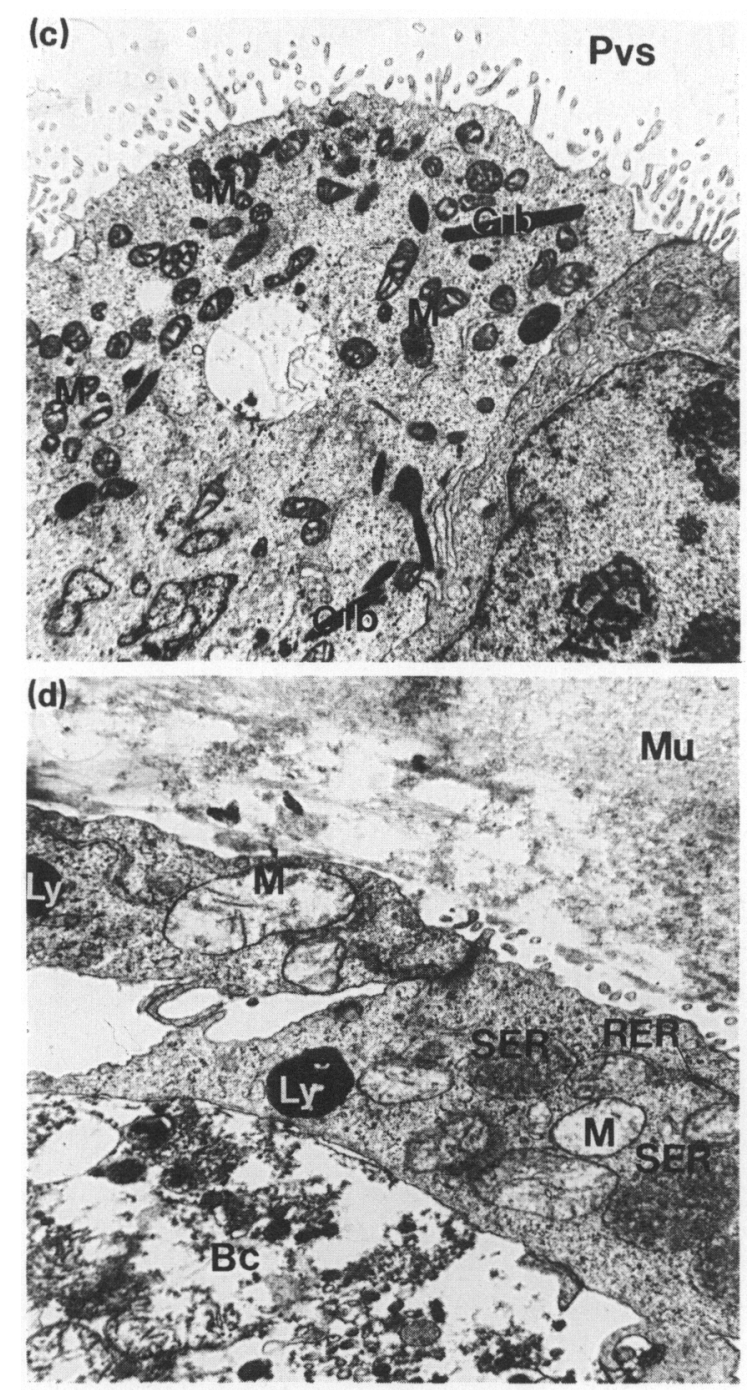

Fig. 5. (c) After transfer into a Day-5 pseudopregnant recipient. Impressive features within the cytoplasm are ovoid to elongated mitochondria (M) and crystalloid inclusion bodies (Cib). Microvilli cover the surface of the trophoblast cells towards the perivitelline space (Pvs). $\times 7160$. (d) After transfer into a Day-6 pseudopregnant recipient. Within the trophoblast cells both smooth (SER) and rough endoplasmic reticulum (RER) can be identified. Ultrastructural features indicating cell damage are vacuolated mitochondria $(M)$ with sparce cristae, secondary lysosomes (Ly) and masses of cell remnants and debris extruded into the blastocyst cavity $(\mathrm{Bc})$. $\mathrm{Mu}$, mucoprotein layer. $\times 14555$.

\section{Light and electron microscopy}

Apart from orientation and arrangement of embryonic cells and internal structure, light microscopic examination focussed primarily on the transformation of the extracellular coverings 
and on the differentiation of the extraembryonic endoderm. Despite the limited number of specimens some conclusions can be drawn. The dissolution of the zona pellucida and the differentiation of the endoderm could be accomplished under asynchronous conditions, indicating that these processes do not require synchrony in embryonic and maternal development. In most cases the zona pellucida had disappeared by 2 days after transfer. Only after transfers into non-pregnant/ non-pseudopregnant recipients (Figs 4a, b) did dissolution seem to be delayed. Replacement of the zona pellucida by the neozona (see Denker \& Gerdes, 1979) was not found in any of the transfer arrangements studied. Endoderm differentiation also appeared to be slightly retarded in Day-0 and Day- 2 uteri but was proved in all experimental groups by the 2 nd and 3 rd day after transfer (Figs $4 \mathrm{c}, \mathrm{d}$ ).

The ultrastructure of synchronously transferred embryos did not reveal signs of damage 2 days after transfer (Fig. 5a), confirming that the transfer manipulation per se had not interfered with further development. In contrast, embryos transferred into asynchronously younger (Fig. 5b) or older uteri (Fig. 5d) showed, besides signs of developmental retardation, distinct signs of degeneration such as detached cells and cell remnants in the perivitelline space and, primarily, in the blastocyst cavity at that time. Ultrastructure was disturbed as indicated by swollen Golgi complex, vacuolated mitochondria with reduced cristae, non-homogeneous ribosome distribution in the cytoplasm and an increase in lysosomal structures. Compared with embryos transferred into less advanced uteri the ultrastructure of those recovered from more advanced uteri was more variable. Apart from clearly damaged blastocysts others with almost normal ultrastructure were found. Another difference between these groups was noted regarding crystalloid inclusion bodies (Fig. 5c): these bodies were only detectable in blastocysts derived from synchronous transfers or from transfers into asynchronously older uteri. Therefore, in contrast to other developmental processes such as dissolution of the zona pellucida or differentiation of the extraembryonic endoderm, the formation of crystalloid bodies seems to require a minimal progestational endometrial transformation.

\section{Discussion}

Due to its essential importance for the establishment and maintenance of pregnancy, the mechanisms of synchrony have been intensively investigated since the first description by Chang in 1950 . Most are still unknown but a few principles of action emerge. Rabbit embryos developing under asynchronous uterine conditions can only survive for a limited time, presumably for not longer than 3 days (Tables 2,3 ). The first reaction to the unphysiological development milieu was a retardation in development (Tables 4, 5; Figs. 5b, d). With increasing time spent in an asynchronous uterus the ability of the embryos to compensate for the unphysiological conditions was lost. The marked variation between individual embryos at the 2 nd and 3rd day after transfer (Table 5), however, proved that some embryos survived asynchronous conditions and maintained cell proliferation for that period of time. The regulatory ability therefore differs considerably between individual blastocysts.

Under suboptimal conditions developmental processes in preimplantation embryos are differently affected. Some processes, such as dissolution of the zona pellucida, endoderm formation (Figs 4c, d) or synthesis of crystalloid bodies (Fig. 5c), can be accomplished, others, for example formation of the neozona (Denker \& Gerdes, 1979), cannot. The ability for further preimplantation development and for implantation are differently regulated as reciprocal transfers show. Day-6 rabbit blastocysts transferred into Day-4 uteri can implant (with most fetuses dying during later pregnancy) while Day-4 blastocysts transferred into Day-6 uteri cannot (Chang, 1950). The ability for normal pre- and postimplantation development and for implantation have to be regarded as separate links in a chain of events obligatory for the maintenance of pregnancy. Thus, only some developmental functions may be disturbed to become limiting factors inhibiting total development. 
Clear differences in the tolerance against insufficient developmental conditions exist between cleavage stages and blastocysts (Wilmut \& Sales, 1981; Lawson et al., 1983). The present study confirms differences even between morulae and blastocysts. After asynchronous transfer the rate of degeneration (Table 3) was higher and growth and cell proliferation were lower in blastocysts than in morulae. At 3 days after transfer the diameter had increased $\times 10$ in former morulae, but only $\times 4$ in blastocysts; rises in thymidine incorporation were $\times 38$ and $\times 22$, respectively. These findings indicate that the sensitivity towards unphysiological conditions increases during preimplantation development. Morgan et al. (1987) emphasize for the pig that blastocysts must attain a minimal degree of maturity to be able to respond to alterations in the uterine environment. This advancement in development, however, is also linked with a higher vulnerability towards suboptimal milieu conditions.

In the present study unphysiological high numbers of embryos were transferred to the uteri of the recipients (see 'Materials \& Methods'). This approach is valid if preimplantation development is analysed. Adams (1960) showed that overcrowding results in embryonic mortality primarily during the post-implantation period.

A characteristic feature of embryonic preimplantation development seems to be the direct involvement of maternal factors. Blastocyst development could be modulated by the actual uterine environment. After 1 day in an asynchronous uterus, blastocysts adapted to the surrounding milieu, as shown by an increased thymidine incorporation by blastocysts recovered from more advanced uteri and a depressed incorporation in the ones recovered from less advanced uteri (Table 5). This finding confirms earlier ones in the sheep (Wilmut \& Sales, 1981; Lawson et al., 1983). Some aspects merit attention. First, Wilmut et al. (1988) reported that the stimulating effect exerted by an asynchronous uterus on retarded sheep embryos was more pronounced than the retarding effect on advanced embryos. Secondly, earlier findings (Wilmut et al., 1985, 1986) indicated that the enhancement of development caused by exposure to a more advanced uterus may be considered an aberrant development rather than a functional adjustment to the present uterine environment because transfer of such embryos resulted in a high incidence of embryonic mortality. This view is supported by experiments involving the simultaneous transfer of synchronous and asynchronous embryos (Wilmut \& Sales, 1981). In this arrangement guaranteeing normal luteal function in the recipient sheep, development of the asynchronously transferred embryos failed, demonstrating the insufficiency of an adequate adaptation of embryo development to the changed uterine milieu.

Findings in the rabbit (Beier et al., 1972; Adams, 1973; Beier, 1973, 1974, 1982) and in the sheep (Lawson \& Cahill, 1983; Vincent et al., 1986) showed conclusively that it was not the chronological age but the actual endometrial transformation that must coincide with the developmental stage of the embryo to guarantee further development. In the studies cited the endometrial transformation and secretion had been changed by exogenous steroid hormones. A comparable modulation of maternal uterine transformation and secretion induced by asynchronous embryos does not seem possible. For example, more advanced blastocysts were not able to delay uterine transformation in order to establish synchrony. The less differentiated embryo therefore seems more flexible and adaptable, at least for a limited period of time, than the differentiated endometrial tissue with its complex interactions between epithelial and stromal cells. Also, the endometrium may be unable to disrupt a programme that has started or may be refractory to mistimed embryonic signals. A different reactivity of embryonic development and endometrial transformation therefore emerges as a principle of synchrony.

Synchrony between embryonic development and the maternal reproductive system is characterized by long- and short-acting interactions. These interactions may be mediated by regulatory signals from the endometrium and/or by quantitative and qualitative changes in nutrients. Missing maternal stimulation by short-term signals is suggested as being responsible for basically the same development of embryos cultured with synchronous or asynchronous uterine flushings $(P>0.05$; Fig. 3). Apart from other reasons (see Fischer, 1988) the loss of co-ordinating maternal stimuli 
seems to be the major deficiency for in-vitro culture of preimplantation embryos. The asynchronous uterus may still provide some appropriate stimuli, as the generally better development of asynchronously transferred embryos compared with cultured ones indicates (Fig. 3). Although the present results give only circumstantial evidence, it seems reasonable to conclude that synchrony is probably generated by a permanent reciprocal interference and cellular modulation of embryonic and maternal development involving exchange of multiple and proper signals.

I thank Professor Henning M. Beier for encouragement and many helpful discussions during the research as well as for valuable comments on the manuscript; Dr Ulla Mootz for performing the transfer operations; Dr Thomas Jung for statistical analyses; and Susanne Echterhagen, Sabine Gülpen, Sabina Hennes, Ria Becht, Jutta Jacobs and Gisela Mathieu for expert technical help. This work was supported by a grant from the Deutsche Forschungsgemeinschaft within the research programme on "Biologie und Klinik der Reproduktion" (Fi 306/1-3).

\section{References}

Adams, C.E. (1960) Prenatal mortality in the rabbit Oryctolagus cuniculus J. Reprod. Fert. 1, 36-44.

Adams, C.E. (1973) Asynchronous egg transfer in the rabbit. J. Reprod. Fert. 35, 613-614.

Beier, H.M. (1973) Die hormonelle Steuerung der Uterussekretion und frühen Embryonalentwicklung des Kaninchens. Habilitationschrift, Medizinische Fakultät, Kiel.

Beier, H.M. (1974) Oviducal and uterine fluids. $J$. Reprod. Fert. 37, 221-237.

Beier, H.M. (1982) Uteroglobin and other endometrial proteins: biochemistry and biological significance in beginning pregnancy. In Proteins and Steroids in Early Pregnancy, pp. 39-71. Eds H. M. Beier \& P. Karlson. Springer-Verlag, Berlin.

Beier, H.M., Mootz, U. \& Kühnel, W. (1972) Asynchrone Eitransplantationen während der verzögerten Uterussekretion beim Kaninchen. Proc. 7 th Int. Congr. Anim. Reprod. \& A.I., München, 3, 1891-1896.

Chang, M.C. (1950) Development and fate of transferred rabbit ova or blastocyst in relation to the ovulation time of recipients. J. exp. Zool. 114, 197-216.

Denker, H.-W. \& Gerdes, H.-J (1979) The dynamic structure of rabbit blastocyst coverings. I. Transformation during regular preimplantation development. Anat. Embryol. 157, $15-34$.

Fischer, B. (1987) Development retardation in cultured preimplantation rabbit embryos. J. Reprod. Fert. 79, 115-123.

Fischer, B. (1988) Embryonalentwicklung in vitro und in vivo. Charakterisierung entwicklungsspezifischer Strukturen und Lebensäußerungen von Präimplantationsembryonen in vitro und in vivo. Copythek, Enke Verlag, Stuttgart.

Fischer, B. \& Meuser-Odenkirchen, G. (1988) A two year follow-up of effects of biotechniques on reproduction in the domestic rabbit, Oryctolagus cuniculus. Lab. Anim. 22, 5-15.

Ham, R.G. (1963) An improved nutrient solution for diploid chinese hamster and human cell lines. Expl Cell Res. 29, 515-526.

Jung, T. \& Fischer, B. (1988) Correlation between blastocyst diameter and DNA or protein synthetic activity in rabbit blastocysts. Biol. Reprod. 39, 1111-1116.
Lawson, R.A.S. \& Cahill, L.P. (1983) Modification of the embryo-maternal relationship in ewes by progesterone treatment early in the oestrous cycle. J. Reprod. Fert. 67, 473-475.

Lawson, R.A.S., Parr, R.A. \& Cahill, L.P. (1983) Evidence for maternal control of blastocyst growth after synchronous transfer of embryos to the uterus of the ewe. J. Reprod. Fert. 67, 477-483.

Maurer, R.R. (1978) Advances in rabbit embryo culture. In Methods in Mammalian Reproduction, pp. 259272. Ed. J. C. Daniel, Jr. Academic Press, New York.

Merchant, H. (1970) Ultrastructural changes in preimplantation rabbit embryos. Cytologia 35, 319-334.

Morgan, G.L., Geisert, R.D., Zavy, M.T., Shawley, R.V. \& Fazleabas, A.T. (1987) Development of pig blastocysts in a uterine environment advanced by exogenous oestrogen. J. Reprod. Fert. 80, 125-131.

Sachs, L. (1984) Applied Statistics. Springer-Verlag, Berlin.

Schumacher, A. \& Fischer, B. (1988) Influence of visible light and room temperature on cell proliferation in preimplantation rabbit embryos. J. Reprod. Fert. 84, 197-204.

Vincent, D.L., Meredith, S. \& Inskeep, E.K. (1986). Advancement of uterine secretion of prostaglandin E2 by treatment with progesterone and transfer of asynchronous embryos. Endocrinology 119, 527-529.

Wilmut, I. \& Sales, D.I. (1981) Effect of an asynchronous environment on embryonic development in sheep. $J$. Reprod. Fert. 61, 179-184.

Wilmut, I., Sales, D.I. \& Ashworth, C.J. (1985) The influence of variation in embryo stage and maternal hormone profiles on embryo survival in farm animals. Theriogenology 23, 107-119.

Wilmut, I., Sales, D.I. \& Ashworth, C.J. (1986) Maternal and embryonic factors associated with prenatal loss in mammals. J. Reprod. Fert. 76, 851-864.

Wilmut, I., Ashworth, C.J., Springbett, A.J. \& Sales, D.I. (1988) Effect of variation in embryo stage on the establishment of pregnancy, and embryo survival and growth in ewes with two embryos. J. Reprod. Fert. 83, 233-237. 